\title{
Splittings and the finite model property
}

\author{
Marcus Kracht \\ II. Department of Mathematics \\ Arnimallee 3 \\ 1000 Berlin 33 \\ GERMANY
}

January 30, 2008

\begin{abstract}
An old and conjecture of modal logics states that every splitting of the major systems K4, S4 and Grz has the finite model property. In this paper we will prove that all iterated splittings of $\mathbf{G}$ have fmp, whereas in the other cases we will give explicit counterexamples. We also introduce a proof technique which will give a positive answer for large classes of splitting frames. The proof works by establishing a rather strong property of these splitting frames namely that they preserve the finite model property in the following sense. Whenever an extension $\Lambda$ has fmp so does the splitting $\Lambda / f$ of $\Lambda$ by $f$. Although we will also see that this method has its limitations because there are frames lacking this property, it has several desirable side effects. For example, properties such as compactness, decidability and others can be shown to be preserved in a similar way and effective bounds for the size of models can be given. Moreover, all methods and proofs are constructive.
\end{abstract}




\section{Introduction}

An old problem of modal logic is to prove that all splittings of $\mathbf{K} \mathbf{4}$ or other important systems have the finite model property (fmp). Up to now this problem has withstood all attempts to prove or disprove it. The only general result to my knowledge is [Blok, 1978] where it is shown that all logics which are iterated splittings of $\mathbf{K}$ have fmp. Unfortunately, this result does not cover any significant logics and is therefore only of theoretical value.

The problem as stated is ambiguous in three ways. There are weaker and stronger readings of it and the stronger versions of this problem will be solved here. There is one reading that says that given a major system $\Lambda(\mathbf{K} \mathbf{4}, \mathbf{S 4}, \mathbf{G}$, Grz) any splitting $\Lambda / f$ has fmp. A slightly more interesting conjecture is that all iterated splittings $\Lambda / F=\bigsqcup\langle\Lambda / f: f \in F\rangle$ have fmp. These are, I guess, the most popular interpretations. But there is a natural question as to whether the base system $\Lambda$ plays a significant role. Of course it is in general false that $\Lambda / f$ has fmp (just take $\Lambda$ without fmp and $f \notin \operatorname{Fr}(\Lambda)$ ); but suppose that $\Lambda$ itself had fmp, does it then hold for $\Lambda / f$ as well? If so, $f$ is said to preserve fmp. We will see that the conjecture that all frames preserve fmp is false, but that a significant class of frames do preserve fmp-though only on the condition that $\Lambda$ contains either of the above mentioned logics. We will also see that there is a splitting Grz/ $N$ of Grz by finitely many frames lacking fmp.

The results proved are obtained by a method that is of considerable interest since it allows to show much more than just preservation of fmp. It can with minor modifications be used to show preservation results for other properties such as compactness, completeness and decidability. Moreover, as the method is constructive it not only proves fmp constructively for a lot of extensions of K4 but it also allows to give a priori bounds for the size of models and thus allows to generate complexity results for the logics as well. Hitherto, only tableau methods had all these properties, but they existed only for a few standard logics (see [Rautenberg, 1983]). Now it seems at least in principle possible to redo all completeness proofs in modal logic by using this method. In fact, in [Kracht, 2001] it is shown that subframe logics can be handled in this way and that the splitting logics S4.Dum and Grz also preserve fmp. Recently, in [Kracht and Wolter, 1991] the same methods were successfully applied to polymodal logics.

I am grateful to Kit Fine for spending a lot of time discussing this essay with me and for providing me with counterexamples. In addition, I want to thank the Studienstiftung des deutschen Volkes for funding while I was at the Centre for Cognitive Science in Edinburgh in 87 as well as Prof. Rautenberg for awakening 
my interest in splittings and for his support.

\section{Basic definitions}

\subsection{Frames and models}

The language $\mathcal{L}$ of modal logic consists of a denumerable set Var of variables, whose elements are denoted by lower case Latin letters, the classical connectives $\wedge, \vee, \neg, \rightarrow$ and $\square, \diamond$. Formulas are denoted by upper case Latin letters such as $P, Q, \ldots$ A (normal) modal logic is a subset $\Theta$ of $\mathcal{L}$ which contains all classical truths, $\mathrm{BD}: \square(p \rightarrow q) \rightarrow(\square p \rightarrow \square q)$ and which is closed under substitution, modus ponens and $\mathrm{MN}: p / \square p$. We reserve upper case Greek letters for logics. A substitution is a mapping $S: Y \rightarrow \mathcal{L}, Y \subseteq$ Var. The effect of $S$ on a formula $Q$ is denoted by $Q[S(p) / p: p \in Y]$ or simply by $Q[S]$. A frame is a pair $f=\langle f, \triangleleft\rangle$ where $f$ is a set and $\triangleleft$ is a relation on $f$. A frame is not assumed to be generated by a single point (or rooted) unless explicitly stated. A p-morphism is a mapping $\pi: f \rightarrow g$ such that $\forall s, t \in f: s \triangleleft t \Rightarrow \pi(s) \triangleleft \pi(t)$ and $\forall s \in f \forall t \in g \exists u \in f:$ $\pi(s) \triangleleft t \Rightarrow t=\pi(u)$. In writing $\pi: f \rightarrow g$ from now on we imply that $\pi$ is a pmorphism. If $\pi$ is injective we write $\pi: f \longmapsto g$ and call $f$ a generated subframe of $g$. If $\pi$ is surjective, $g$ is called a contraction of $f$, in symbols $\pi: f \rightarrow g$. For a finite frame $g$ we say that $g$ omits $f$ if $f$ is not the contraction image of a generated subframe of $g$. A valuation on $f$ is a mapping $\beta: Y \rightarrow 2^{f}, Y \subseteq$ Var. $\beta$ uniquely extends to $\bar{\beta}: \mathcal{L}(Y) \rightarrow 2^{f}$. The pair $\langle f, \beta\rangle$ is called a model. It is said to be finite if $f$ is finite and finitely generated if $Y$ is finite. Generally, frames are assumed to be finite throughout this paper. If $s \in f$ then $\langle f, \beta, s\rangle \vDash Q$ iff $s \in \bar{\beta}(Q)$ and $\langle f, \beta\rangle \vDash Q$ iff $\bar{\beta}(Q)=f$. Finally, $f \vDash Q$ iff $\forall \beta: Y \rightarrow 2^{f}:\langle f, \beta\rangle \vDash Q$. The logic $L f$ of $f$ is the set of all $Q$ such that $f \vDash Q$. $f$ is a $\Theta$-frame if $L f \supseteq \Theta$. $\operatorname{Fr}(\Theta)\left(\operatorname{Fr}_{f}(\Theta)\right)$ denotes the collection of all (finite) $\Theta$-frames, and $\operatorname{Md}(\Theta)\left(\operatorname{Md}_{f}(\Theta)\right)$ the collection of all (finite) $\Theta$-models i.e. models $\langle f, \beta\rangle$ where $f$ is a (finite) $\Theta$-frame. $\langle f, \beta\rangle$ is called refined if $\forall r, s \in f \exists Q \in \mathcal{L}: r \neq s \Rightarrow\langle f, \beta, r\rangle \vDash Q$ and $\langle f, \beta, s\rangle \not \notin Q$. This can be reformulated as follows: call a p-morphism $\pi: f \rightarrow g$ admissible for $\beta$ if $\forall t \in g \forall r, s \in \pi^{-1}(t) \forall p \in Y:\langle f, \beta, r\rangle \vDash p$ iff $\langle f, \beta, s\rangle \vDash p$. Then $\langle f, \beta\rangle$ is refined iff every admissible $\pi: f \rightarrow g$ is injective. If $\langle f, \beta\rangle$ is not refined, there is a uniquely defined p-morphism $p: f \rightarrow f / \beta$ which makes the structure $\langle f / \beta, \gamma\rangle$ with $\beta(q)=p^{-1}(\gamma(q))$ refined. We call it the refined equivalent of $f$.

We say, $\langle f, \beta\rangle$ is a model for $X, X \subseteq \mathcal{L}$ iff $\exists s \in f:\langle f, \beta, s\rangle \vDash Q$ for all $Q \in X$. We also call $\langle f, \beta, s\rangle$ a model for $X$, where $\langle f, \beta, s\rangle \vDash Q$ for all $Q \in X$. As $f$ does 
not have to be one generated, $s$ does not need to generate $f$, nor does $s$ need to be initial in $f$. A model for $X$ is called minimal if it is refined, $\beta: \operatorname{var}(X) \rightarrow 2^{f}$ and no model for $X$ is based on a p-morphic image of a generated subframe of $f$. If $\langle f, \beta\rangle$ is a minimal model for $X$, then $f$ is generated by a single point $s$ for which $\langle f, \beta, s\rangle \vDash Q$ for all $Q \in X$.

\subsection{The proof method}

Definition 1 Let $\Lambda$ be a modal logic. $\Theta_{1}$ is said to split $\Lambda$ if a logic $\Theta_{2}$ exists such that for all $\Gamma \supseteq \Lambda$ either $\Gamma \subseteq \Theta_{1}$ or $\Gamma \supseteq \Theta_{2}$. If $\Theta_{1}$ splits $\Lambda, \Theta_{2}$ is uniquely determined and denoted by $\Lambda / \Theta_{1}$.

If $\Lambda$ has fmp then $\Theta$ splits $\Lambda$ only if it is the logic of a finite frame generated by a single point, or, equivalently, if it is the logic of a finite, subdirect irreducible (fsi) algebra. It has been shown by [Blok, 1978] that this is not a sufficient condition. For example, the logic of the frame consisting of a single reflexive point meets that condition but does not split $\mathbf{K}$. For a general investigation into splittings see [Kracht, 1989].

Definition 2 Let $\Theta$ be a logic. $f$ is called a splitting frame if its logic Lf splits $\Theta$. We write $\Theta / f$ instead of $\Theta / L f$. If $f$ is finite and generated by $s, \Theta / f=\Theta\left(\Sigma_{f}\right)$ where $\Sigma_{f}=\square^{(n)} \Delta(f) \rightarrow \neg p_{s}$ for some $n \in \omega$ and

$$
\begin{aligned}
\Delta(f)= & \bigwedge\left\langle p_{u} \rightarrow \neg p_{v}: u \neq v\right\rangle \\
& \wedge \bigwedge\left\langle p_{u} \rightarrow \diamond p_{v}: u \triangleleft v\right\rangle \\
& \wedge \bigwedge\left\langle p_{u} \rightarrow \neg \diamond p_{v}: u \Varangle v\right\rangle \\
& \wedge \bigvee\left\langle p_{u}: u \in f\right\rangle
\end{aligned}
$$

where $u, v$ range over $f$. We used the convention $\square^{(n)} p:=\bigwedge\left\langle\square^{i} p: 0 \leq i \leq n\right\rangle$. In case $\Theta \supseteq K 4 n$ can be chosen to be 1 .

We give a sketch why a finite frame for $\Theta\left(\Sigma_{f}\right)$ omits $f$. For if $g$ is such that a generated subframe $h$ of $g$ maps p-morphically onto $f$, a valuation $\beta$ into $g$ can be found such that $\beta, g, t \vDash \square^{(n)} \Delta(f) \wedge \neg p_{s}$ for some $t \in g$ and thus $\Sigma_{f} \notin L g$.

Definition 3 Let $\Theta$ be a logic and $f$ be a splitting frame for $\Theta . \quad f$ is said to preserve the finite model property beyond $\Theta$ if for all $\Lambda \supseteq \Theta \Lambda / f=\Lambda \sqcup \Theta / f$ has fmp whenever $\Lambda$ has fmp. 
The way we prove preservation of fmp for a frame is shown in the following scheme: Suppose $X$ is finite and consistent with $\Lambda / f$. Then a finite set $X^{\sharp}$ of formulas of type $\square^{(m)} \Sigma_{f}\left[Q_{s} / p_{s}: s \in f\right]$ is added to $X$. Since $X^{\sharp} \subseteq \Lambda / f, X ; X^{\sharp}:=$ $X \cup X^{\sharp}$ is consistent with $\Lambda / f$ and hence with $\Lambda$. Thus there is a finite $\Lambda$-model $\langle g, \beta, s\rangle$ for $X ; X^{\sharp}$. If it is minimal, $g$ is a $\Lambda / f$-frame. Thus we have reduced the problem to finding appropriate constraints $X^{\sharp}$ or to finding suitable substitutions $Q: f \rightarrow \mathcal{L}$, which we call nets for $f$. For suppose that $g$ does not omit $f$. Then we have a subframe $i: h \longmapsto g$ and a contraction $p: h \rightarrow f$ and if all models $\langle f, \beta\rangle,\langle g, \gamma\rangle,\langle h, \eta\rangle, \eta(p)=\gamma(p) \cap h$, are refined, there is a set $\left\{Q_{s}: s \in f\right\}$ of formulas such that $\langle h, \eta, t\rangle \vDash Q_{r}$ iff $p(t)=s$. Thus if $t_{0}$ generates $h$ then $s_{0}=p\left(t_{0}\right)$ generates $f$ and so $\left\langle h, \eta, t_{0}\right\rangle \vDash \square^{(n)} \Delta(f) \wedge p_{s_{0}}[Q]\left(=\neg \Sigma_{f}[Q]\right)$. Hence, for a suitable $m,\left\langle g, \gamma, r_{0}\right\rangle \vDash \diamond^{(m)} \neg \Sigma_{f}[Q]$, where $r_{0}$ generates $g$ and so, if we include $\square^{(m)} \Sigma_{f}[Q]$ in $X^{\sharp},\langle g, \gamma\rangle$ can no longer be a model for $X ; X^{\sharp}$.

\section{Contexts and Recognizable Frames}

\subsection{Nets and Contexts}

As usual, the modal degree $\operatorname{dg}(P)$ is defined as the maximal nesting of modalities in $P$. We summarize the last section in the following definition.

Definition 4 A pair $\langle p, i\rangle$ of p-morphisms is a context for $f$ in $g$ iff $i: h \mapsto g$ and $p: h \rightarrow f$ for some $h$. An indexed set of formulas $Q: f \rightarrow \mathcal{L}$ is a net for $f$. $Q$ recognizes $f$ in the context $\langle p, i\rangle$ if for all $\beta$ such that $\langle g, \beta\rangle$ is refined and for all $t \in h:\langle g, \beta, i(t)\rangle \vDash Q_{s}$ iff $p(t)=s$. We define the degree of $Q$ by $\operatorname{dg}(Q):=$ $\max \left\{\operatorname{dg}\left(Q_{s}\right): s \in f\right\}$ and $\operatorname{var}(Q):=\bigcup\left\langle\operatorname{var}\left(Q_{s}\right): s \in f\right\rangle$. Also we define the substitution of a net for $f$ in a diagram $\Delta(f)$ by $\Delta(f)[Q]:=\Delta(f)\left[Q_{s} / p_{s}: s \in f\right]$ and similarly for the splitting formula. A set of nets for $f$ is a trawl for $f$. If $T$ is a trawl then $\operatorname{dg}(T):=\max \{\operatorname{dg}(Q): Q \in T\} . T$ is called finite if $\operatorname{dg}(T)$ is finite. $T$ recognizes $f$ in the context if one of its nets recognize $f$ in that context.

Thus a finite frame $g$ omits $f$ if there is no context for $f$ in $g$.

Definition $5 f$ is strongly recognizable $(=s . r$.$) in a class of frames \mathfrak{X}$ if there is a finite trawl which recognizes $f$ in every context out of $\mathfrak{X}$. $f$ is weakly recognizable $(=w . r$.$) in \mathfrak{X}$ if there is a finite trawl such that for every model $\langle g, \beta\rangle$ with $g$ in $\mathfrak{X}$ such that $g$ does not omit $f$ there is a context for $f$ in $g$ in which $f$ is recognized by that trawl. 
$\Theta$ is m-transitive if $\square^{(m)} q \rightarrow \square^{(m+1)} q \in \Theta$.

Theorem 6 If $f$ is w.r. in $\operatorname{Md}_{f}(\Theta)$ and $\Theta$ is m-transitive then $f$ preserves fmp beyond $\Theta$.

Proof. Let $T$ be a finite trawl that recognizes $f$ in $\operatorname{Md}_{f}(\Theta)$. Let $X$ be $\Lambda / f$ consistent. Following our proof scheme we have to design an appropriate $X^{\sharp}$. We define $X^{\sharp}:=\left\{\square^{(m)} \Sigma(f)[Q]: Q \in T, \operatorname{var}(Q) \subseteq \operatorname{var}(X)\right\} . X^{\sharp}$ is finite since the trawl of nets based on the variables of $X$ is finite. Clearly this trawl recognizes $f$ in $\operatorname{Md}_{f}(\Theta)$. Now let $\langle g, \beta\rangle$ be a minimal model for $X ; X^{\sharp}$ and let $s$ generate $g$. Then if $g$ does not omit $f$ there is a context $\langle p, i\rangle$ such that $\forall t \in h:\langle g, \beta, i(t)\rangle \vDash Q_{s}$ iff $p(t)=s$ and $Q \in T$ is based on $\operatorname{var}(X)$. Now if $t_{0}$ generates $h$ and $p\left(t_{0}\right)=s_{0}$ we have $\left\langle g, \beta, i\left(t_{0}\right)\right\rangle \vDash \square^{(p)} \Delta(f) \wedge Q_{s_{0}}(=\neg \Sigma(f)[Q])$; but on the other hand, since $g$ is generated by $s$ and $\langle g, \beta, s\rangle \vDash \square^{(m)} \Sigma(f)[Q]$ we have $\left\langle g, \beta, i\left(t_{0}\right)\right\rangle \vDash \Sigma(f)[Q]$ which is a contradiction. Therefore $g$ omits $f$ and $f$ preserves fmp beyond $\Theta$.

Finally we present a class of frames which preserve fmp for every weakly transitive $\Theta$, namely the cycle free frames. A frame $f$ is called cycle free iff $\neg \exists s \in f: s \triangleleft^{\star} s$, where $\triangleleft^{\star}$ is the transitive closure of $\triangleleft$.

Theorem 7 Let $\Theta$ be any logic and $f \in \operatorname{Fr}_{f}(\Theta)$ be finite and cycle free then $f$ is s.r. in $\mathrm{Fr}_{f}(\Theta)$. In addition, if $\Theta$ is weakly transitive, $f$ preserves fmp.

Proof. We only have to show the first part. Let $\langle p, i\rangle$ be a context for $f$ in $g$. Since $f$ is cycle free there is a number $n \in \omega$ such that $f \models \square^{(n)} 0 . \operatorname{dp}(f)$ is defined to be the least number with that property. Since $p: h \rightarrow f$ is surjective $\operatorname{dp}(h)=\operatorname{dp}(f)<\omega$. Thus the trawl of nets of degree less or equal to $\operatorname{dp}(f)$ recognizes $f$ in $\langle p, i\rangle$.

\subsection{State Descriptions}

Let $\beta: Y \rightarrow 2^{g}$ be a valuation from a finite set $Y$ into $g$. Then the state description at $_{s}$ of $s \in g$ is defined by at ${ }_{s}:=\bigwedge\langle p: p \in Y, s \in \beta(p)\rangle \wedge \wedge\langle\neg p: p \in Y, s \notin \beta(p)\rangle$. For a finite subset $N$ of $g$ the state description is defined by at ${ }_{N}:=\bigvee\left\langle\mathrm{at}_{s}: s \in N\right\rangle$. In addition, let at ${ }_{Y}$ denote the set of all possible state descriptions from $Y$, i.e. at $_{Y}=\left\{P_{C}: C \subseteq Y\right\}$ with $P_{C}=\wedge\langle p: p \in C\rangle \wedge \wedge\langle\neg p: p \in Y-C\rangle$. Given a finite set $X$ of formulas, the extended state description $A t_{s}$ of $s$ is defined by $A t_{s}:=\bigwedge\langle Q: Q \in \operatorname{sf}(X), s, \beta \vDash Q\rangle \wedge \wedge\langle\neg Q: Q \in \operatorname{sf}(X), s, \beta \not \vDash Q\rangle$ where $\operatorname{sf}(X)$ is the set of subformulas of the formulas in $X$. 


\subsection{Definable sets and properties}

Our method for proving preservation crucially depends on definability in $\Theta$-frames. Let us therefore investigate some questions on definability in K4-frames before we prove conservation property for K4-frames. However, let us first recall some notions and facts about $\mathbf{K 4}$-frames. A set $C$ of points in a $\mathbf{K 4}$-frame is called a cluster if either $C=\{t\}$ and $t \nless t$ or $C$ is maximal such that $C \times C \subseteq \triangleleft$. In the former case $C$ is called improper. In a K4-frame clusters behave exactly like points and so we write $C \triangleleft D$ or $C \triangleleft t$ whenever $C$ sees $D$ or $t$. A point is called terminal or of depth 1 if $\forall t^{\prime}: t \triangleleft t^{\prime} \Rightarrow t^{\prime} \triangleleft t$. Likewise a cluster is called terminal if all of its points are terminals. A points $t$ or cluster is of depth $n+1$ iff for all $t^{\prime}$ with $t \triangleleft t^{\prime} \pitchfork t, t^{\prime}$ is of depth $\leq n$ and there is at least one such $t^{\prime}$ of depth $=n$.

Let $f$ be a frame, $N \subseteq f$ a subset. $N$ is called definable if there is a number $n$ such that for every valuation $\beta: X \rightarrow 2^{f}$ such that $X$ is finite and $\langle f, \beta\rangle$ is refined a formula $Q^{\beta}$ of degree $\leq n$ exists satisfying $\langle s, f, \beta\rangle \vDash Q^{\beta}$ iff $s \in N$. In this case we also say that $N$ is $n$-definable and that $Q^{\beta}$ defines $N$ in $f$ with respect to $\beta$. A property is $(n)$-definable in a class $\Omega$ of frames if for every $f \in \mathfrak{R}$ the set of points which have that property is $(n)$-definable. It is easy to see that the class of $n$-definable properties is closed under all boolean operations and likewise the class of $n$-definable subsets. A first nontrivial result is that the property 'terminal' (= 'of depth 1') is 2-definable in the class of $\mathbf{K 4}$-frames and thus also the property 'nonterminal'. To see this, let $\beta: X \rightarrow 2^{f}$ be such that $\langle f, \beta\rangle$ is refined and let $t$ be a point of depth 1 . Then $t$ lives in a terminal cluster $C$. Now define $Q_{t}$ by

$$
Q_{t}= \begin{cases}\mathrm{at}_{t} \wedge \square 0 & \text { if } t \nless t \\ \mathrm{at}_{t} \wedge \diamond 1 \wedge \square\left(\mathrm{at}_{C} \wedge \wedge\left\langle\diamond \mathrm{at}_{t^{\prime}}: t^{\prime} \in C\right\rangle\right) & \text { else }\end{cases}
$$

$Q_{t}$ defines exactly $\{t\}$. Consequently, any finite set of points of depth 1 is 2definable. But as $X$ is finite, there are only finitely many of them and so all sets of terminals are 2-definable. Thus, 'terminal', which corresponds to the set of all terminal points, is 2-definable; and so is its complement. To be explicit, if $D_{n}$ denotes the defining formula for the set of points of depth $n$, then we have

$$
\begin{aligned}
D_{1}= & \bigvee\left\langle A \wedge \square 0: A \in \mathrm{at}_{Y}\right\rangle \\
& \vee \bigvee\left\langle B \wedge \square\left(\bigvee_{C \in S} C \wedge \bigwedge_{D \in S} \diamond D: S \subseteq \mathrm{at}_{Y}, B \in S\right\rangle\right.
\end{aligned}
$$

To show that all points of depth $\mathrm{k}, k>1$, are also definable, we will make use of the fact that if $N$ is an $m$-definable subset and $\mathcal{P}$ an $n$-definable property, the 
set of points which have $\mathcal{P}$ within $N$ is $m+n$-definable. In order to state this properly, let $f$ be a frame and $g \subseteq f$ a subset of points, not necessarily generated. Then call $\left\langle g, \triangleleft_{f} \cap g^{2}\right\rangle$ a subframe of $f$ (cf. [Fine, 1985]). If $\beta: X \rightarrow 2^{f}$ is a valuation, then a unique valuation onto $g$ is defined by restricting the values to $g$, which we denote by $\beta$ as well. Now suppose that there is a formula $Q$ such that $\forall t \in f:\langle f, \beta, t\rangle \vDash Q \Leftrightarrow t \in g$. Then define the localization of a formula $P$ onto $g$, in symbols $P \downarrow Q$, via

$$
\begin{array}{lll}
P \downarrow Q & =P \wedge Q & \text { if } P \text { is nonmodal } \\
\left(P_{1} \wedge P_{2}\right) \downarrow Q & =\left(P_{1} \downarrow Q\right) \wedge\left(P_{2} \downarrow Q\right) & \\
(\neg P) \downarrow Q & =Q \wedge \neg(P \downarrow Q) & \\
(\square P) \downarrow Q & =Q \wedge \square(Q . \rightarrow . P \downarrow Q) & \\
(\diamond P) \downarrow Q & =Q \wedge \diamond(Q . \wedge . P \downarrow Q)
\end{array}
$$

By induction one can show

Proposition 8 (Localization) If $g \subseteq f$ is a subframe and $Q$ a formula such that $\langle f, \beta, s\rangle \vDash Q \Leftrightarrow s \in g$ for some valuation $\beta$, then $\langle f, \beta, t\rangle \vDash P \downarrow Q$ iff $t \in g$ and $\langle g, \beta, t\rangle \vDash P$. In addition $\operatorname{dg}(P \downarrow Q)=\operatorname{dg}(Q)+\operatorname{dg}(P)$.

We include the warning here that even if $\langle f, \beta\rangle$ is refined, $\langle g, \beta\rangle$ need not be refined. This generally happens only when $g$ is a generated subframe of $f$.

Now we will construct formulas defining the singletons $\{t\}$ for each $t$ of finite depth. We will do this by induction on the depth of $t$ and in addition we will get the formulas $D_{k}$. Let us therefore suppose that such formulas have been built for $\operatorname{dp}(t), k<n$. Let then $t$ be any point of depth $\geq n$. Following [Fine, 1985] we define the width $w d(t)$ of $t$ by $w d(t)=\left\{\mathrm{at}_{s}: \mathrm{dp}(s) \geq n, t \triangleleft s\right\}$ and the $\operatorname{span} \mathrm{sp}(t)$ of $t$ by $\operatorname{sp}(t)=\{s: \operatorname{dp}(s)<n, t \triangleleft s\}$. Say that $t$ is of minimal width if no successor of depth $\geq n$ has lesser width, and say that $t$ is of minimal span if no successor of depth $\geq n$ has lesser span. Then in a refined frame, $t$ is of depth $n$ exactly if it is of minimal width and minimal span. This characterization allows a stepwise construction of $Q_{t}$. Using localization, we can define the property 'is of minimal width w' with $\mathfrak{w} \subseteq$ at $_{Y}$ by

$$
W_{\mathfrak{w}}=\square(\bigvee\langle A: A \in \mathfrak{w}\rangle) \wedge \bigwedge\langle\square \diamond B: B \in \mathfrak{w}\rangle . \downarrow\left[\bigwedge\left\langle\neg D_{i}: i \in n\right\rangle\right]
$$

To define the property 'is of minimal span t', with $t$ any set of points of depth $<n$, note that in general if $\mathfrak{s} \subseteq f$ is a set of points and $Q_{x}$ defines $\{x\}$ for $x \in \mathfrak{s}$ with respect to $\beta$, then for $\mathrm{t} \subseteq \mathfrak{s} P_{\mathrm{t}}:=\wedge\left\langle\diamond Q_{x}: x \in \mathrm{t}\right\rangle \wedge \wedge\left\langle\neg \diamond Q_{x}: x \notin \mathrm{t}\right\rangle$ defines the 
set $A_{\mathrm{t}}:=\{t \in f: \forall x \in \mathfrak{s}: t \triangleleft x \Leftrightarrow x \in \mathrm{t}\}$. So, $P_{\mathrm{t}}$ defines 'is of span $\mathrm{t}$ ', if $\mathfrak{s}$ is the set of points of depth $<n$. And the formula

$$
S_{\mathrm{t}}=P_{\mathrm{t}} \wedge \square \diamond P_{\mathrm{t}} \cdot \downarrow\left[\bigwedge\left\langle\neg D_{i}: i \in n\right\rangle\right]
$$

defines 'is of minimal span t'. Thus

$$
Q_{t}=\mathrm{at}_{t} \wedge W_{w d(t)} \wedge S_{\mathrm{sp}(t)}
$$

defines exactly $\{t\}$. Then $D_{n}$ is the disjunction of all possible formulas of this type.

\section{Extensions of $\mathrm{K} 4$}

\subsection{Classifying the extensions of K4}

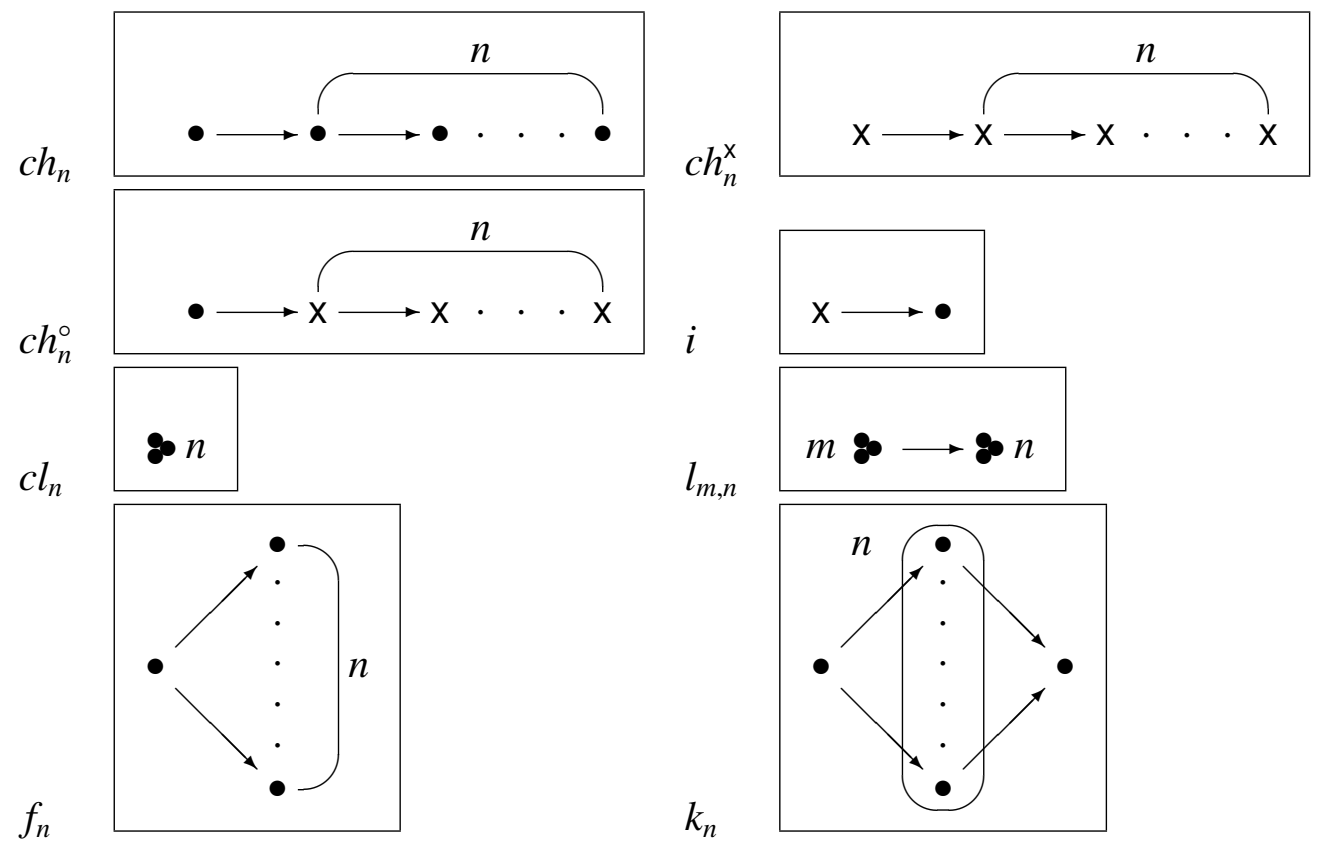




\begin{tabular}{|c|c|c|c|c|}
\hline \multirow[t]{2}{*}{ axiom } & \multicolumn{2}{|c|}{ name } & \multicolumn{2}{|c|}{ rep. over $K 4$} \\
\hline & $L / S$ & P.D. & splittings & subframes \\
\hline$\square p \rightarrow \diamond p$ & $\mathbf{D}$ & D4 & $c h_{0}^{\mathrm{X}}$ & none \\
\hline$\square p \rightarrow p$ & $\mathbf{T}$ & S4 & $c h_{0}^{\mathrm{x}}, i$ & $c h_{0}^{\mathrm{X}}$ \\
\hline$\square(\square p \rightarrow p) \rightarrow \square p$ & $\mathbf{W}$ & $\mathbf{G}$ & none & $c h_{0}$ \\
\hline$\diamond p \wedge \diamond q \rightarrow \diamond(p \wedge q) . \vee$ & & & & \\
\hline \multirow{4}{*}{$\diamond(p \wedge \diamond q) . \vee . \diamond(\diamond p \wedge q)$} & \multirow{4}{*}{$\begin{array}{l}\mathbf{H} \\
\mathbf{I}_{n} \\
\mathbf{J}_{n}\end{array}$} & \multirow{4}{*}{$\begin{array}{l}\text { K4.3 } \\
\text { K4.I. }_{n} \\
\text { K4.J }_{n}\end{array}$} & none & $f_{2}^{\square}$ \\
\hline & & & none & $f_{n}^{\square}$ \\
\hline & & & none & $c h_{n}^{\square}$ \\
\hline & & & \multicolumn{2}{|c|}{ over $G$} \\
\hline \multirow{3}{*}{$\begin{array}{l}\square 0 \\
\square^{n} 0\end{array}$} & \multirow[t]{3}{*}{$\mathbf{V}$} & & $c h_{1}^{\mathrm{X}}$ & $c h_{1}^{\mathrm{X}}$ \\
\hline & & & $c h_{n}^{\mathrm{x}}$ & $c h_{n}^{\mathrm{x}}$ \\
\hline & & & \multicolumn{2}{|c|}{ over S4 } \\
\hline \multirow{2}{*}{$\begin{array}{l}p \leftrightarrow \square p \\
p \rightarrow \square \diamond p\end{array}$} & \multicolumn{2}{|l|}{$\operatorname{Tr}$} & $c h_{1}, c l_{2}$ & $c h_{1}, c l_{2}$ \\
\hline & B & S5 & $c h_{1}$ & $c h_{1}$ \\
\hline$\square \diamond p \rightarrow \diamond \square p$ & $\mathbf{M}$ & S4.1 & $c l_{2}$ & none \\
\hline$\diamond \square p \rightarrow \square \diamond p$ & $\mathbf{G}$ & S4.2 & $f_{2}$ & none \\
\hline \multirow{5}{*}{$\begin{array}{l}\diamond p \wedge \diamond q \rightarrow \diamond(p \wedge q) \vee \vee \\
\diamond(p \wedge \diamond q) . \vee \diamond(\diamond p \wedge q) \\
\square(\square(p \rightarrow \square p) \rightarrow p) \rightarrow p \\
\square(\square(p \rightarrow \square p) \rightarrow p) \wedge \diamond \square p \rightarrow p\end{array}$} & & & & \\
\hline & $\mathbf{H}$ & $\mathbf{S 4 . 3}$ & $f_{2}, k_{2}$ & $f_{2}$ \\
\hline & Grz & Grz & $c l_{2}, l_{2,1}$ & $\mathrm{cl}_{2}$ \\
\hline & Dum & S4.4 & $l_{2,1}$ & none \\
\hline & & $\mathbf{S} \mathbf{4}_{n}$ & $c h_{n}$ & $c h_{n}$ \\
\hline
\end{tabular}

The above list contains almost all important axioms for logics beyond K4. The axioms $I_{n}$ and $J_{n}$ are somewhat more complex but their geometrical meaning is rather easy to state. $I_{n}$ excludes that a point has $n+1$ distinct incomparable successors and $J_{n}$ excludes that there is a strictly ascending chain of more than $n$ points. We will see that $I_{n}$ has a splitting representation over $\mathbf{S 4}$. There are two main ways of naming logics. The column $L / S$ gives the name of the axiom as proposed by Lemmon and Scott. The column P.D. gives the 'trivial' name for the extension defined by the axiom in question. (As in chemistry, there is a systematic catalogue of names and some small number of trivial names for logics which are commonly used. The L/S notation is now becoming more popular for obvious reasons.) A splitting representation of an axiom $Q$ over a logic $\Theta$ is a finite set $N$ of frames such that $\Lambda(Q)=\Lambda / N$ whenever $\Lambda \supseteq \Theta$. A subframe representation of $Q$ over $\Theta$ is a finite set $N$ such that $\Lambda(Q)=\Lambda_{N}$ for $\Lambda \supseteq \Theta$. For the latter we refer to [Fine, 1985]. If $f=\langle f, \triangleleft\rangle$ is a frame $f^{\square}$ is the set of all frames $g=\langle f, \bar{\triangleleft}\rangle$ such that $\triangleleft \cup i d_{f}=\bar{\triangleleft} \cup i d_{f}$. 


\subsection{Frames Preserving fmp beyond K4}

Call a cluster $C$ meager if $\operatorname{card}(C)=1$ and call a frame meager iff every cluster is meager.

Definition 9 Let $f$ be a frame and $\mathfrak{I}$ the set of terminal clusters of $f . \quad f$ is called solid if for all clusters $C, D C=D$ iff $\forall S \in \mathfrak{I}: C \triangleleft S \Leftrightarrow D \triangleleft S)$. In other words, points that see the same set of terminals belong to the same cluster.

Lemma 10 A meager and solid frame is strongly recognizable in $\operatorname{Md}_{f}(\boldsymbol{K 4})$.

Proof. Let $\langle p, i\rangle$ be a context for $f$ and $\mathfrak{I}$ the set of terminal clusters (=points) of $f$. For $\{t\} \in \mathfrak{I}$ put $Q_{t}:=p_{t} \wedge \square 0$ if $t \nless t$ and $Q_{t}:=p_{t} \wedge \square p_{t}$ if $t \triangleleft t$. We then define $Q_{[s]}:=\bigwedge\left\langle\diamond Q_{t} \mid\{t\} \in \mathfrak{I}, s \triangleleft t\right\rangle \wedge \wedge\left\langle\neg \diamond Q_{t} \mid\{t\} \in \mathfrak{I}, s \nless t\right\rangle . Q: s \longrightarrow Q_{[s]}$ is a net for $f$. Now $p$ is completely determined by the value of the terminals in h. Thus if $\eta: Y \rightarrow 2^{h}$ is a finite valuation we have to look for formulas based on $Y$ which are exactly true at a given set of terminal clusters of $h$. If $C$ is a terminal cluster $\left.\rho_{C}:=a t_{C} \wedge \square a t_{C} \wedge \wedge\left\langle\square \diamond a t_{t} \mid t \in C\right\rangle\right)$ if $C$ is proper and $\rho_{C}:=a t_{C} \wedge \square 0$ if $C$ is improper is exactly true at $C$ since we assume $\langle h, \eta\rangle$ to be refined. If $X_{s}$ is the set of terminal clusters mapped to $s$ then $\rho_{s}:=\bigvee\left\langle\rho_{C} \mid C \in X_{s}\right\rangle$ is true exactly at $X_{s}$. Thus $Q\left[\rho_{s} / p_{s}\right]$ is a net of degree 4 which recognizes $f$ in the context $\langle p, i\rangle$. We have shown that $f$ is strongly recognizable in $\operatorname{Md}_{f}(\mathbf{K 4})$.

Corollary 11 All $f_{n}, n>1$, preserve fmp beyond $\mathbf{K} 4$.

Lemma $12 c l_{n}$ is weakly recognizable for $n>1$ in $\operatorname{Md}_{f}(\boldsymbol{K} 4)$.

Proof. $c l_{n}=\langle n, \triangleleft\rangle, \triangleleft=n \times n$. Define $Q_{i}:=p_{i} \wedge \square \diamond p_{i}$. A context for $c l_{n}$ can always be chosen for a given $g$ such that $p=i d_{h}$. Then clearly $T:=\left\{Q\left[\alpha_{i} / p_{i}\right] \mid\right.$ $\forall i \in n: \alpha_{i}=\bigwedge\langle p \mid p \in S\rangle \wedge \bigwedge\langle\neg p \mid p \notin S\rangle, S \subseteq Y, Y$ finite $\}$ is a trawl for $c l_{n}$ which recognizes it in every context of type $\left\langle i d_{h}, i\right\rangle$. Thus $c l_{n}$ is w.r. in $\operatorname{Md}_{f}(\mathbf{K 4})$.

Lemma 13 Let $e$ be strongly recognizable in $\operatorname{Md}_{f}(\mathbf{K 4})$ and $q: f \rightarrow e$ a $p$ morphism such that every fibre is a cluster of $f$. Then $f$ is weakly recognizable in $\operatorname{Md}_{f}(\boldsymbol{K} 4)$.

Proof. Let $\langle p, i\rangle$ be a context for $f$ in $g$, let $\langle g, \beta\rangle$ be refined. Then $\langle q \circ p, i\rangle$ is a context for $e$ in $g$ and thus there is a net $N: e \rightarrow \mathcal{L}$ of bounded degree which recognizes $e$ in that context. Now let $s \in e$ and define $f_{s}:=q^{-1}(s)$ and 
$h_{s}:=p^{-1}\left[f_{s}\right]$. Then $f_{s}$ is a cluster and the restrictions $p_{s}:=p \uparrow h_{s}$ and $q_{s}:=q \uparrow f_{s}$ are p-morphisms. Moreover, if $\widetilde{p}$ is such that all $\widetilde{p}_{s}: h_{s} \rightarrow f_{s}$ are p-morphisms and $q \circ \widetilde{p}$ is a p-morphism, then $\widetilde{p}$ is a p-morphism. For if $x \triangleleft y$ in $h$ then $q \circ \widetilde{p}(x) \triangleleft q \circ \widetilde{p}(y)$ and thus $\widetilde{p}(x) \triangleleft \widetilde{p}(y)$ since all the fibres of $q$ are clusters. And if $\widetilde{p}(x) \triangleleft y$ then $q \circ \widetilde{p}(x) \triangleleft q(y)$; whence there is a $u \in h$ with $q \circ \widetilde{p}(x) \triangleleft q \circ \widetilde{p}(u)$ and again we have $\widetilde{p}(x) \triangleleft \widetilde{p}(u)$. This fact allows a piecemeal construction of a $\widetilde{p}$ such that $\langle\widetilde{p}, i\rangle$ is a context in which $f$ can be recognized.

First we let $\widetilde{p}$ be such that $q \circ \widetilde{p}=q \circ p$ and hence we only have to specify $\widetilde{p}_{s}$ for every $s \in e$. Now take $\left\langle h_{s}, \beta\right\rangle$. This frame need not be refined. However, as $p_{s}: h_{s} \rightarrow f_{s}$ and $f_{s} \cong c l_{n}$ for some $n \in \omega$, all terminal clusters of $h_{s}$ are of size $\geq n$ and this is true also for the refined equivalent of $h_{s}, h_{s} / \beta$. Hence there is a $\widetilde{p}_{s}$ which factors through $h_{s} \rightarrow h_{s} / \beta$. Moreover, it can be chosen so that all nonterminal points of $h_{s} / \beta$ are mapped onto a single point. This concludes the definition of $\widetilde{p}_{s}$. All the fibres can be defined by formulas of degree $\leq 2$. Thus if $t \in f_{s}$ and $Q_{t}$ defines $\widetilde{p}_{s}^{-1}(t)$ in $h_{s}$, then $Q_{t}^{s} \downarrow N_{s}$ defines $\widetilde{p}^{-1}(t)$ in $h$, by localization. Thus if $T$ is a trawl of degree $k$ recognizing $e$ in every context, then the trawl of all nets of type $Q_{t}^{s} \downarrow N_{s}$ is a trawl of degree $k+2$ weakly recognizing $f$ in $\operatorname{Md}_{f}(\mathbf{K 4})$.

Corollary 14 A solid frame preserves fmp beyond $\mathbf{K} 4$.

For the formulation of the next theorem, let $g \subseteq f$ be a subframe; then $f-g$ denotes the complement subframe of $g$ in $f$ consisting of all points not in $g$.

Theorem 15 Let $f$ be a frame, $r \mapsto f$ a (generated) subframe which is cycle free. If $f-r$ is solid then $f$ is weakly recognizable in $\operatorname{Md}_{f}(\boldsymbol{K} 4)$.

Proof. Due to Lemma 13 it suffices to show that $f$ is strongly recognizable if $f$ is meager and $f-r$ solid. Let therefore be $\langle p, i\rangle$ be a context for $f$ in $g$ and $f$ be a meager and $f-r$ solid frame. Let $\langle g, \beta\rangle$ be refined. Now if $s \in r$, then $p^{-1}(s)$ is of depth $\leq \operatorname{card}(r)$, and so there is a number $k$ such that every point of $p^{-1}[r]$ is $k$-definable. Consequently, the terminal points of $h-p^{-1}[r]$ are $k+2$-definable. Define a valuation $\gamma: Y \rightarrow 2^{h}$ where $Y=\left\{p_{t} \mid t\right.$ is terminal in $\left.h-p^{-1}[r]\right\}$ by $\gamma\left(p_{t}\right)=\{t\} .\left\langle h-p^{-1}[r], \gamma\right\rangle$ is refined and thus there is a net $N: f \rightarrow \mathcal{L}$ recognizing $f-r$ in $\left\langle h-p^{-1}[r], \gamma\right\rangle$ in the context $\langle p 1 h-r, i 1 g-r\rangle$. Then if $Q_{t}$ defines $\{t\}$ in $h, R$ with $R_{s}=N_{s}\left[Q_{t} / p_{t}\right]$ recognizes $f$ in $\langle p, i\rangle$. 


\subsection{Application of the results}

\subsubsection{Extensions of $\mathbf{G}$}

Corollary 16 Every $\mathbf{G}$-frame preserves fmp beyond $\mathbf{K} \mathbf{4}$ and in particular also beyond $\boldsymbol{G}$. Hence all $\boldsymbol{G}_{n}=\boldsymbol{G} /$ ch $_{n}^{\times}$have fmp.

This result follows from Theorem 7. It is worth noting that since this theorem was comparatively easy to prove we cannot expect it to be very powerful. In fact, splitting out $f$ in the lattice of extensions of $\mathbf{G}$ yields a weaker logic than splitting out $f^{\bullet}$, the reflexive counterpart of $f$, in the extension lattice of $\mathbf{S 4}$. For example $\mathbf{S 4 . 3}$ results from $\mathbf{S} 4$ by splitting out two frames but $\mathbf{G . 3}$ is not a splitting logic of G. It can be shown that $\mathbf{G . 3}$ cannot even be obtained by splitting out infinitely many frames of $\mathbf{G}$ even though $\mathbf{G . 3}$ is a subframe logic. From the fact that $c h_{k}^{\circ}$ all preserve fmp we deduce that there is an ascending chain of logics $\mathbf{K} \mathbf{4}_{n}:=\mathbf{K} 4 /\left\{c h_{k}^{\circ} \mid k \in n\right\}$ which have fmp. It is easily seen that $\mathbf{K 4}_{\omega}:=\lim \left\{\mathbf{K} \mathbf{4}_{n}:\right.$ $n \in \omega\}$ has the same finite models as $\mathbf{G}$ (cf. [Rautenberg, 1979]) but since $\mathbf{K} \mathbf{4}_{\omega}$ is not finitely axiomatisable, $\mathbf{K} \mathbf{4}_{\omega} \neq \mathbf{G}$. Consequently, $\mathbf{K} \mathbf{4}_{\omega}$ does not have fmp. It is thus disproved that all splittings $\mathbf{K 4} / F$ with $F$ a set of transitive frames has fmp. However, if we want to have an answer for finite $F$, we have to be more sophisticated (see below). Using the fact that the algebras of $c h_{n}^{\circ}$ are 0-generated algebras we can deduce with the help of the splitting theorem in [Kracht, 1990] that $K \mathbf{4}_{\omega}$ is a constant extension of $K \mathbf{4}$ and likewise that $K \mathbf{4}_{\omega} . \mathbf{3}$ is a constant extension of $\mathbf{K 4 . 3}$; moreover, it can be shown that $\mathbf{K} \mathbf{4}_{\omega} . \mathbf{3}$ has the same constant theorems as $\mathbf{G . 3}$ and that $\mathbf{K} \mathbf{4}_{\omega} . \mathbf{3}$ is meet irreducible in the lattice of normal modal logics with cover G.3 ([Kracht, 1991]).

\subsubsection{Extensions of S4}

As we will see in the next section a result as strong as the one for $\mathbf{G}$ cannot hold for S4. But collecting what we have proved so far we get

Corollary 17 Every $\boldsymbol{S 4}$-frame of depth $\leq 2$ with the exception of $l_{m, n}$ preserves fmp beyond $\mathbf{K} \mathbf{4}$ and consequently also beyond $\mathbf{S} \mathbf{4}$

We can do a little bit better than that:

Lemma $18 l_{1, n}$ preserves fmp beyond $\mathbf{S} 4$.

Sketch of Proof. A S4-context $\langle p, i\rangle$ for $l_{1, n}$ can always be chosen so that $p^{-1}\left(l_{1, n}\right)$ is of depth 2 and generated by a point. We know that there is a formula $Q$ of 
degree 3 which is true exactly at the terminals of $p^{-1}\left(l_{1, n}\right)$ and thus $\neg Q$ is true exactly at the nonterminals. But the nonterminals are exactly the points that are mapped onto the generating point of $l_{1, n}$.

Given that we know that $\mathbf{S 4}$ and Grz have fmp (and that this is even constructively shown via tableau methods) we have the following results which incidentally are now also constructively proved (we will return to the issue of constructivity at the end of this essay).

Corollary 19 S4.1, S4.2 and S5 have fmp.

Corollary 20 Grz.1 and Grz.2 have fmp.

It is perhaps instructive to see a concrete example which might show how easy completeness proofs are using our method.

Proposition $21 \Theta_{1}:=\Theta / c h_{1}$ has fmp if $\Theta$ has fmp, for $\Theta \supseteq$ S4. In particular since $\mathbf{S} \mathbf{4}$ has fmp, $\mathbf{S} \mathbf{5}$ has fmp, too.

Proof. Let $P$ be consistent with $\Theta_{1}$. Since $P \rightarrow \square \diamond P \in \Theta_{1}, P$; $\square \diamond P$ is consistent with $\Theta_{1}$ and a fortiori consistent with $\Theta$ and hence it has a finite model. A minimal model for $P ; \square \diamond P$ is easily seen to be a cluster and hence omits $c h_{1}$. It is therefore a $\Theta_{1}$-model.

With the exception of the kites $k_{n}$, the $l_{m, n}$ for $m>1$ and the chains $c h_{n}$ we have proved the preservation property for all the frames mentioned in the beginning. But for the chains there is nothing to show for Maximova has proved in [Maksimova, 1975] that any logic containing $\mathbf{S} \mathbf{4}_{n}=\mathbf{S 4} / \mathrm{ch}_{n}$ has fmp and consequently all chains have the preservation property. However, it also follows easily from the fact that points of depth $\leq n$ are $k$-definable for some $k$. The rest of the frames still remain a problem. As regards $l_{m, n}$, they can be shown to preserve fmp using a more sophisticated proof method involving extended state descriptions, which we will not describe here. Instead we refer to [Kracht, 1990]. The same applies to the kite $k_{2}$. For the other kites we have found no way to prove the conservation property. Our personal guess is that $k_{n}$ fails to preserve fmp for $n>2$.

\subsection{Some counterexamples}

We have already seen that there are logics $\mathbf{K} 4 / F$ lacking fmp. Now we are giving counterexamples to show that not all frames preserve fmp beyond K4. The first 
example was pointed out to me by Kit Fine. Take $\Theta:=\mathbf{S 4 . I _ { 3 } . 2}$. S4.I $\mathbf{I}_{3}$ is the logic of S4-frames of width 3. As is known from [Fine, 1985], S4. $\mathbf{I}_{3}$ has fmp and the results of the previous section then show fmp for $\Theta$. Now in [Kracht, 1990] it is shown that $\Lambda:=\Theta /\left\{d_{1}, d_{2}, f_{1}, f_{2}\right\}$ does not have fmp.
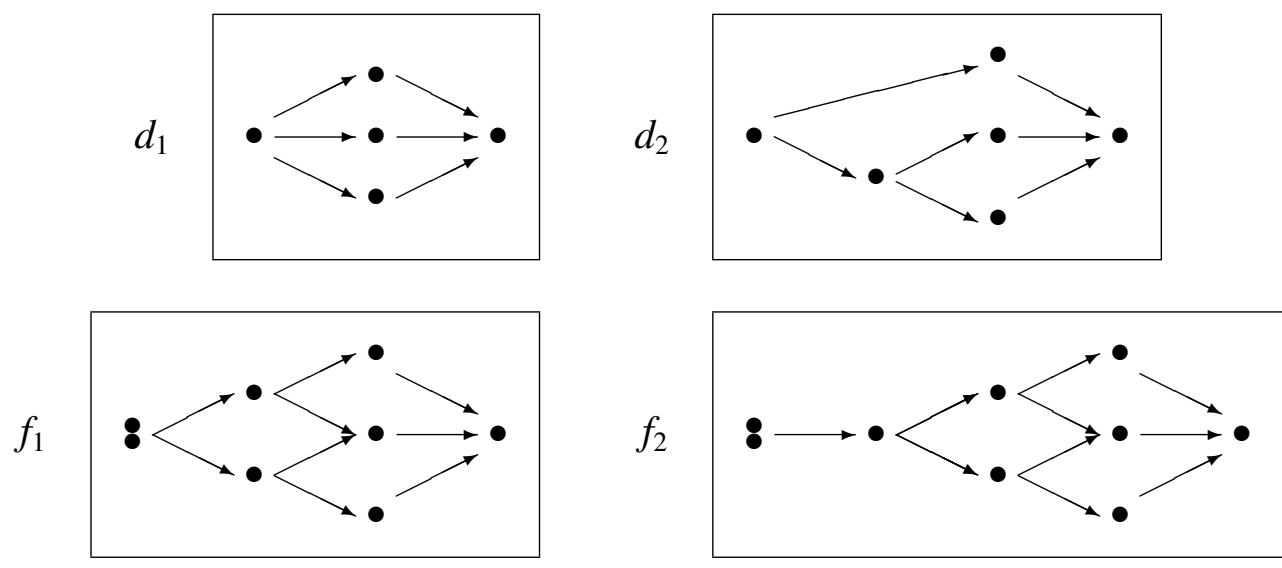

Thus at least one of the above frames fails to preserve fmp beyond S4. We have not investigated the question which of these frames is the culprit. However, it is possible to give an even better example and thereby disprove a conjecture that was given to me by A. Wroński that all iterated splittings of Grz have fmp. This is then a direct counterexample for $\mathbf{S 4}$ and $K 4$ as $\mathbf{G r z}$ and $\mathbf{S 4}$ are splittings of $\mathrm{K4}$. Of course, no such example can exist for $\mathbf{G}$ as we have proved. The counterexample is the logic of the following frame.

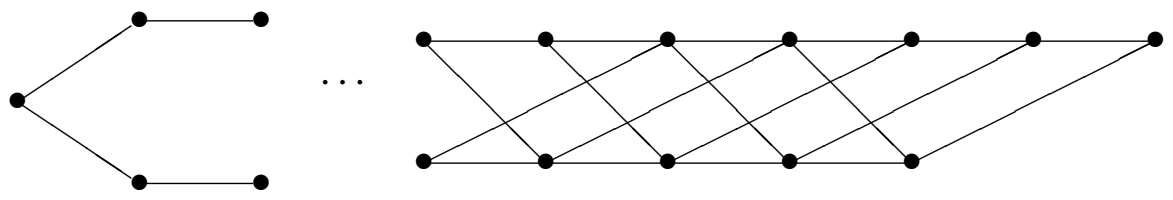

Let me quote from [Kracht, 1991] that this logic is axiomatized over Grz.2 by adding two more splitting axioms and the subframe axioms for the frames given below. Moreover, it lacks fmp while all proper extensions have fmp. All that needs to be done here is to show that the subframe axioms can be replaced by finitely many splitting axioms. We risk a conjecture here that for extensions of $\mathbf{S 4}$ and especially of Grz this is always possible. Thus any axiomatization of extensions of $\mathbf{S 4}$ by means of subframe axioms could be seen as shorthand for some more complicated splitting axiomatization. The relevant special cases of this conjecture will be proved here (though not in all detail). 


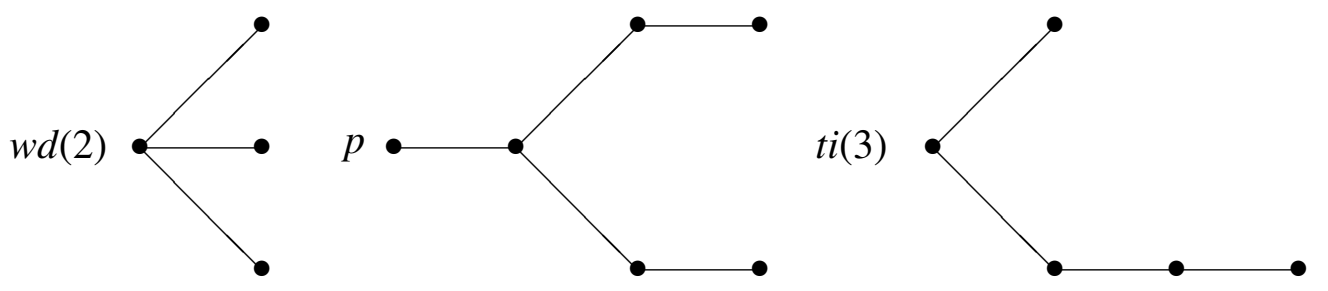

Proposition $22 S_{4} I_{n}=S 4 / N$ where $N$ is the set of all frames of cardinality $\leq 2^{n+1}$ which are not of width $n$.

Proof. It is clear that $\mathbf{S 4} / N \leq \mathbf{S 4} \mathbf{I}_{n}$. The converse remains to be established. We prove this in the particular case $n=2$ but nothing depends on that. We look at the weak canonical model $\mathbb{C}$ for $\mathbf{S 4} / N$ and show that it is of width 2 , by which the claim follows. (Recall [Fine, 1974] for the necessary details.) Suppose that in $\mathbb{C}$ there is a point $a$ with three incomparable successors $t, u, v$. At first we have to distinguish whether or not $t$ resp. $u$ or $v$ are of depth 1 or not. This opens eight possibilities which can be treated alike. Let us assume that $t$ is of depth 1 while $u$ and $v$ are not. There are then formulae $T, U, V$ such that $t \in T, u \in U$ and $v \in V$. (To save ink we confuse formulae with their value in $\mathfrak{C}^{\text {.) }}$ ) Moreover, we can arrange it that if $x$ is a strict successor of $u$ (i.e. $u \triangleleft x$ but $x \nless u$ ) then $x \notin U$ and likewise that $x \notin V$ if $x$ is a strict successor of $v$. Now set $Z=\neg \diamond T \wedge \neg \diamond U \wedge \neg \diamond V$. Then $Z$ contains all strict successors of $U$ and $V$ and some more points incomparable to $t, u$ and $v$. If we allow ourselves to write $U \triangleleft Z$ for $(\forall u \in U)(\exists z \in Z)(u \triangleleft z)$ then we have $U \triangleleft Z, V \triangleleft Z$. Now we define $A=\diamond T \wedge \diamond U \wedge \diamond V, B=\diamond T \wedge \diamond U \wedge \neg \diamond V$, $C=\diamond T \wedge \neg \diamond U \wedge \diamond V$ and $D=\neg \diamond T \wedge \diamond U \wedge \diamond V$. We then have the following frame

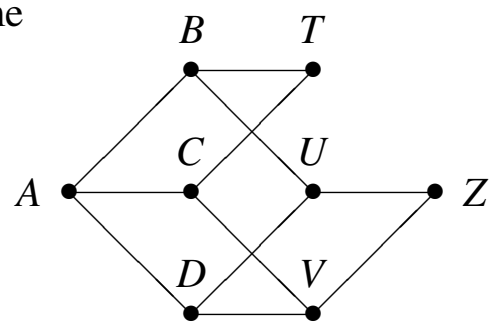

Here $X \rightarrow Y$ iff $X \triangleleft Y$. If no arrow exists from $X$ to $Y$ we have $(\forall x \in X)(\forall y \in$ $Y)(x \nless y)$. It may be the case that some of the sets depicted above are empty in which case they are dropped from the picture. Now if we take the subframe generated by $a \in A$ then the picture shows a p-morphism from this frame onto a frame with at most eight points violating $\mathbf{I}_{3}$.

Using similar arguments we can prove the following proposition. To state it we use the convention $f \otimes g$ to denote the frame $\left\langle f \cup g, \triangleleft_{f} \cup \triangleleft_{g} \triangleleft f \times g\right\rangle$. Intuitively, this frame results from placing $f$ before $g$. 
Proposition $23 \boldsymbol{S 4}_{t i(3)}=\boldsymbol{S} \boldsymbol{4} /\{t i(3), t i(3) \ominus \bullet\} . S 4_{\bullet \ominus p}=\boldsymbol{S 4} /\{p, p \ominus \bullet\}$.

\section{Preservation of fmp and constructive reduction}

It should be stressed that weak or strong recognizability in some classes of models establishes much more than just preservation of fmp. It can be seen as a semantic tool to derive a general property which I will call constructive reduction for $r$. The idea is the following. Given a logic $\Lambda$ and an axiom $P$ we know that $(\ddagger) \vdash_{\Lambda(P)} Q \Leftrightarrow$ $\left(\exists Q^{\sharp} \subseteq_{\text {fin }} \Lambda(P)\right) Q^{\sharp} \vdash_{\Lambda} Q$, or equivalently, $\vdash_{\Lambda(P)} Q \Leftrightarrow\left(\exists Q^{\sharp} \in \Lambda(P)\right) \vdash_{\Lambda} Q^{\sharp} \rightarrow Q$. However, we know of this equivalence only via classical logic because it is only after having given a proof for $Q$ in $\Lambda(P)$ we can name this formula (or finite set) $Q^{\sharp}$. Thus this equivalence is not constructively valid because we have no means to establish $Q^{\sharp}$ beforehand. And so, an effective reduction of provability in $\Lambda(P)$ to provability in $\Lambda$ via ( $\$)$ is impossible unless we have a computable function $(-)^{\sharp}: Q \mapsto Q^{\sharp}$. Thus we can in some sense say that we property of $P$ that we desire is that ( $\$)$ is constructively true. Whenever it is we say that $P$ admits constructive reduction for $\vdash$ with respect to $\Lambda$. It is immediate that constructive reduction for $\vdash$ is equivalent to preservation of decidability.

Weak recognizability is one way to establish for a splitting logic $\Theta / f$ that it allows for constructive reduction from $\Lambda / f$ to $\Lambda$ for every $\Lambda \supseteq \Theta$. Unfortunately, it works on two conditions, namely that $\Theta$ is weakly transitive and that $\Lambda$ is complete for the class of frames $\mathfrak{X}$ in which $f$ is weakly recognizable. Let us state this explicitly.

Proposition 24 Suppose that $f$ is weakly recognizable in a class $\mathfrak{X}$ of (generalized) frames which is closed under taking generated subframes and contractions. Then a constructive reduction from $\Lambda / f$ to $f$ is possible if $\Lambda$ is $\mathfrak{X}$-complete and weakly transitive.

Proof. Define (-) $)^{\sharp}$ as in the proof of Theorem 6. Then $\vdash_{\Lambda / f} P \Leftrightarrow \models_{M d(\Lambda / f) \cap \mathfrak{x}} P^{\sharp} \rightarrow$ $P \Leftrightarrow \models_{M d(\Lambda) \cap \mathfrak{X}} P^{\sharp} \rightarrow P \Leftrightarrow \vdash_{\Lambda} P^{\sharp} \rightarrow P$.

In brackets we have deliberately added the qualification 'generalized' because nothing changes if we replace Kripke-frames by generalized frames; and in order to be truly general one has to include generalized frames at this point. It is clear that $\operatorname{Fr}(\Lambda)$ is then taken to be the class of generalized frames of $\Lambda$. The first and the last of the equivalences is conditional on completeness whereas the middle equivalence holds only for weakly transitive logics. In order to get the best possible reduction result for $f$ one has to find the largest class in which it is weakly 
recognizable. To name just one example, it is possible to show that cycle-free frames are weakly recognizable in the class of differentiated generalized frames, whence a constructive reduction via ( $\$)$ is possible if only weak transitivity holds. A last point concerns the closure under generated subframes and contractions. Of course, by the way things are proved we expect such a condition because we could not conclude $\mathfrak{X}$-completeness of $\Lambda / f$. But there are also specific examples to show this, namely the axiom $\mathbf{2}$ which preserves fmp beyond $\mathbf{G r z}$ but not completeness with respect to finite trees.

The assumption of weak transitivity still is a limitation that has to be overcome. Define a new consequence relation $\Vdash$ which extends $\vdash$ by the rule $\phi \Vdash$ $\psi / \phi \Vdash \square \psi$. It is straightforward to see that $\phi \Vdash \psi \Leftrightarrow\left\{\square^{k} \phi: k \in \omega\right\} \vdash \psi$. Say that $\Lambda$ has the global fmp if every consistent set of the type $\left\{\square^{k} \phi: k \in \omega\right\} \cup\{\psi\}$ has a finite model; and say that $\Lambda$ is globally decidable if the problem ' $\phi \Vdash \psi$ ', or equivalently, the problem ' $\left\{\square^{k} \phi: k \in \omega\right\} \vdash_{\Lambda} \psi$ ' is decidable. Then it is possible to show that weak recognizability in $\mathfrak{X}$ shows that a constructive reduction for $\Vdash$ is possible. For suppose that $f$ is weakly recognizable in $\mathfrak{X}$. Then $\vdash_{\Lambda / f} Q \Leftrightarrow\left\{\square^{k} Q^{\sharp}: k \in \omega\right\} \vDash_{M d(\Lambda) \cap \mathfrak{x}} Q \Leftrightarrow\left\{\square^{k} Q^{\sharp}: k \in \omega\right\} \vdash_{\Lambda} Q$; which holds without assuming weak transitivity, $P$ being some theorem of $\Lambda / f$. Now we know that $P$ depends only on the variables of $Q$ and $\operatorname{var}\left(Q^{\sharp}\right) \subseteq \operatorname{var}(Q)$. Thus if we want to reduce the problem $R \Vdash_{\Lambda / f} Q$ the fact that we are faced with infinitely many formulae for which to define some reducing conditions is of no significance. It is easy to see that we have the following reduction: $(\dagger): R \Vdash_{\Lambda / f} Q \Leftrightarrow\left\{\square^{k} R: k \in\right.$ $\omega\} \vdash_{\Lambda / f} Q \Leftrightarrow\left\{\square^{\sharp} R: k \in \omega\right\} \cup\left\{\square^{k}(Q \wedge R)^{\sharp}: k \in \omega\right\} \vdash_{\Lambda} Q \Leftrightarrow R ;(R \wedge Q)^{\sharp} \Vdash Q$. By consequence, if $\Lambda$ is globally decidable then $\Lambda / f$ is globally decidable as well.

Proposition 25 Suppose that $f$ is weakly recognizable in a class $\mathfrak{X}$ which is closed under generated subframes and contractions. Then $f$ admits a constructive reduction for $\Vdash$ for all $\Lambda$ which are $\mathfrak{X}$-complete.

So much about the connection between weak recognizability and constructive reduction. A lot of preservation results now follow immediately. We have mentioned decidability and $\mathfrak{X}$-completeness. We may add that there is also a preservation for $\mathfrak{Y}$-completeness for all classes $\mathfrak{Y} \subseteq \mathfrak{X}$ which are closed under generated subframes and contractions. But there are also stronger notions of completeness namely compactness and weak compactness (see [Fine, 1974]). These notions are suitably relativised to classes of frames. Say that $\Lambda$ is $\mathfrak{X}$-compact if every consistent set has a model based on a $\Lambda$-frame from $\mathfrak{X}$ and say that $\Lambda$ is weakly $\mathfrak{X}$ compact if this holds for all sets based on a finite set of variables. We just have to convince ourselves of the fact that the transition from finite sets to infinite sets is 
harmless and that the restriction to finite variables is also of no significance. The latter rests on the fact that $(-)^{\sharp}$ maps formulae with variables from a set $Y$ into formulae with variables from $Y$. Thus $X$ is $\Lambda / f$-consistent iff $X ; X^{\sharp}$ is $\Lambda$-consistent and moreover $\operatorname{var}(X)=\operatorname{var}\left(X^{\sharp}\right)$. That this behaviour of $(-)^{\sharp}$ is essential is shown by some negative example in [Kracht and Wolter, 1991] where weak compactness is lost under constructive reduction.

Proposition 26 Suppose that $f$ is weakly recognizable in a class $\mathfrak{X}$ closed under generated subframes and contractions. Then $f$ preserves weak $\mathfrak{X}$-compactness and $\mathfrak{X}$-compactness.

The same type of investigation could be carried out for yet another consequence relation $\| \Vdash$ which extends $\Vdash$ by the rule $\phi\|\vdash / \phi\| s \psi$ where $s$ is any substitution. Then $P \|_{\Lambda} Q$ iff $\Lambda(Q) \subseteq \Lambda(P)$. The question of constructive reduction does not arise here since $P \mathbb{H}_{\Lambda / f} Q \Leftrightarrow P ; \Sigma_{f} \mathbb{t}_{\Lambda} Q$. But nevertheless weak recognizability has an effect on the decidability of this consequence which is in general rather strongly undecidable. For if we take $P \| r_{\Lambda} Q$ and let either $P$ or $Q$ be a splitting axiom $\Sigma_{f}$ with $f$ weakly recognizable, then the problem ' $P \| r_{\Lambda} Q$ ' becomes decidable. The argument is rather easy. It is known ([Rautenberg, 1980]) that $Q \|_{\Lambda} \Sigma_{f}$ exactly if $Q \notin L f$ which is decidable since $f$ is finite. And if $f$ is weakly recognizable in $\mathfrak{X}$ then $\Sigma_{f} \| t Q$ is also decidable given that $\Lambda$ is $\mathfrak{X}$-complete and decidable. Finally and most importantly, axiomatization problems ' $\Lambda(P)=\Lambda(Q)$ ' are decidable if, say, $\Lambda(P)=\Lambda / f$ is known.

As a final point I want to mention iterated splittings. The fact that we can in some sense push up properties once of course implies that we can push it up any number of times. For theoretical results this is enough. However, since this method also allows to give effective bounds for models for $\Lambda / f$ in case such bounds are known for $\Lambda$ there is then the concern as to how fast these bounds develop by iteration. For suppose that $N=\left\{f_{i}: i \in n\right\}$ is a set of weakly recognizable frames and that $(-)^{\#_{i}}$ is the reduction function associated with $f_{i}$; then by iterated reduction we get $\vdash_{\Lambda / f} Q \Leftrightarrow\left(\ldots\left(Q^{\sharp_{0}}\right)^{\#_{1}} \ldots\right)^{\sharp_{n-1}} \vdash_{\Lambda} Q$. This could in principle be a rather large set, but since all these functions depend on the variables of the formula only, we have $\left(Q^{\sharp_{i}}\right)^{\sharp_{j}} \leftrightarrow Q^{\sharp_{i}} \wedge Q^{\sharp_{j}}$ and so $\vdash_{\Lambda / N} Q \Leftrightarrow Q^{\sharp_{0}} ; Q^{\sharp_{1}}: \ldots ; Q^{\sharp_{n-1}} \vdash_{\Lambda} Q$. Consequently, if the original function $\ell$ bounding the size of models in $\Lambda$ was exponential in $Q$ we expect a multiplicative behaviour of the iterated splittings in the sense that the bounding function $\ell_{N}$ for $\Lambda / N$ is bounded by the product of the bounding functions $\ell_{i}$ for the $\Lambda / f_{i}$ for we have $\ell_{N}(Q) \leq \ell\left(Q ; Q^{\sharp_{0}} ; \ldots ; Q^{\sharp_{n-1}}\right) \leq \prod_{0}^{n-1} \ell\left(Q^{\sharp_{i}} ; Q\right)=\prod_{0}^{n-1} \ell_{i}(Q)$ - assuming that $\ell$ depends only on the number of subformulae. 


\section{References}

[Blok, 1978] W. J. Blok. On the degree of incompleteness of modal logics. Bulletin of the Section of Logic of the Polish Academy of Sciences, 7:167 - 175, 1978.

[Fine, 1974] Kit Fine. Logics containing K4, Part I. Journal of Symbolic Logic, 39:229-237, 1974.

[Fine, 1985] Kit Fine. Logics containing K4, Part II. Journal of Symbolic Logic, 50:619-651, 1985.

[Kracht and Wolter, 1991] Marcus Kracht and Frank Wolter. Properties of independently axiomatizable bimodal logics. Journal of Symbolic Logic, 56:1469$1485,1991$.

[Kracht, 1989] Marcus Kracht. On the logic of category definitions. Computational Linguistics, 15:111-113, 1989.

[Kracht, 1990] Marcus Kracht. An almost general splitting theorem for modal logic. Studia Logica, 49:455-470, 1990.

[Kracht, 1991] Marcus Kracht. Internal Definability and Completeness in Modal Logic. PhD thesis, FU Berlin, 1991.

[Kracht, 2001] Marcus Kracht. Reducing Modal Consequence Relations. Journal of Logic and Computation, 11:879-907, 2001.

[Maksimova, 1975] Larisa L. Maksimova. Pretabular extensions of Lewis S4. Algebra i Logica, 14:28-55, 1975.

[Rautenberg, 1979] Wolfgang Rautenberg. Klassische und nichtklassische Aussagenlogik. Vieweg, Braunschweig/Wiesbaden, 1979.

[Rautenberg, 1980] Wolfgang Rautenberg. Splitting lattices of logics. Archiv für Mathematische Logik, 20:155-159, 1980.

[Rautenberg, 1983] Wolfgang Rautenberg. Modal tableau calculi and interpolation. Journal of Philosophical Logic, 12:403-423, 1983. 